\title{
REVISIONES
}

\section{Predictores antropogénicos y biofísicos de deforestación en la Amazonía: hacia la integración de actividades REDD+}

\author{
Anthropogenic and biophysical predictors of deforestation in the Amazon: \\ towards integrating REDD+ activities
}

\author{
Pedro Manuel Villa ${ }^{a, b, c *}$, Sebastião Venâncio Martins ${ }^{b, d}$, \\ Silvio Nolasco de Oliveira Neto ${ }^{\text {, }}$, Alice Cristina Rodrigues a,c \\ * Autor de correspondencia: a Universidade Federal de Viçosa, Programa de Pós-Graduação em Botânica, \\ CEP 36570000, Viçosa, Minas Gerais, Brasil, tel.: 55-31-83134545, pedro.villa@ufv.br \\ ${ }^{\mathrm{b}}$ Universidade Federal de Viçosa, Departamento de Engenharia Florestal, Laboratório de Restauração Florestal, \\ Viçosa, Minas Gerais, Brasil. \\ ${ }^{\mathrm{c}}$ Fundación para la Conservación de la Biodiversidad, 7101, Puerto Ayacucho, estado Amazonas, Venezuela. \\ ${ }^{\mathrm{d}}$ Universidade Federal de Viçosa, Departamento de Engenharia Florestal, Viçosa, Minas Gerais, Brasil.
}

\begin{abstract}
SUMMARY
The Amazon is one of the most important tropical forests in the world, providing important goods and services to society. However, these forests are threatened by land use change. Deforestation and forest degradation are the two drivers that significantly contribute to both carbon emissions to the atmosphere and loss of biodiversity. Although there are alternatives to reduce emissions from deforestation and forest degradation, there is still little research on methods for assessing and monitoring the relationship between these processes and biodiversity (REDD+), as well as the impacts generated by the socioecological system on the stability and recovery of ecosystem services. With this review, we aimed at (i) describing the relationship between biodiversity and ecosystem functioning; (ii) analyzing the possible synergistic impacts of biophysical and anthropogenic predictors of deforestation; and, consequently, (iii) proposing the integrated implementation of REDD+ strategies in the Amazon basin. To increase REDD+ efficiency in the Amazon, it is essential to simultaneously evaluate the socioecological and biophysical predictors that directly affect the current and future demands for ecosystem goods and services. Accordingly, it is important to estimate the magnitude of multifactorial effects of deforestation predictors for the integration of REDD+ actions. Such estimations should be made through the rehabilitation of degraded lands with successional and permanent agroforestry systems, as well as with the application of different restoration methods.
\end{abstract}

Key words: agroforestry, biomass, carbon storage, forest restoration, land use change.

\section{RESUMEN}

La Amazonía es uno de los bosques tropicales más importantes del mundo, que proporcionan importante bienes y servicios a la humanidad; sin embargo, se encuentra amenazado por los continuos cambios en el uso de la tierra. La deforestación y degradación forestal son las dos razones que contribuyen significativamente con las emisiones de carbono a la atmósfera y pérdida de la biodiversidad. Aunque existen alternativas para reducir las emisiones de carbono por deforestación y degradación forestal evitadas (REDD), aún son pocas las investigaciones sobre métodos de evaluación y monitoreo de la relación de estos procesos con la biodiversidad, así como de los impactos generados por el sistema socioecológico en la estabilidad y recuperación de servicios ecosistémicos. El objetivo de esta revisión consiste en i) describir la relación biodiversidad-función ecosistémica, para ii) analizar los posibles impactos sinérgicos de predictores biofísicos y antropogénicos de la deforestación y degradación, con la finalidad de iii) proponer la implementación integral de estrategias REDD+ en la cuenca Amazónica. Para incrementar la eficiencia REDD+ en la Amazonía, es fundamental evaluar simultáneamente los predictores antropogénicos y biofísicos que repercuten directamente sobre la demanda actual de los bienes y servicios ecosistémicos. Por este motivo, es importante describir la magnitud de los efectos multifactoriales que tienen los predictores de deforestación para la integración de actividades REDD+, a través de la rehabilitación de áreas degradadas con sistemas agroforestales sucesionales y permanentes, así como con la aplicación de diferentes métodos de restauración.

Palabras clave: agroforestería, almacenamiento de carbono, cambio en el uso de la tierra, biomasa, restauración forestal. 


\section{INTRODUCCIÓN}

La cuenca Amazónica es la mayor extensión de bosque tropical continuo con cerca del $25 \%$ de las especies vegetales descritas del mundo, y almacena aproximadamente $50 \%$ del carbono presente en bosques tropicales (Strassburg et al. 2010, Mitchard et al. 2014). La Amazonía es importante por los servicios ecosistémicos que provee, como el mantenimiento de la biodiversidad, regulación bioclimática, y procesos biogeoquímicos (Peres et al. 2010, Strassburg et al. 2010, Aragão et al. 2014). Por esta razón, el estudio de procesos ecosistémicos en Amazonas tienen alta importancia frente a los alarmantes cambios climáticos globales y cambios en el uso de la tierra a escala regional, presentando las mayores tasas de deforestación histórica y acumulada entre los bosques tropicales (Lapola et al. 2013, Aragão et al. 2014). Se destaca que del total de las emisiones de carbono a la atmósfera durante los últimos 10 años, la deforestación es responsable de hasta 47 \% (Aguiar et al. 2016). Por esta razón, existe la preocupante y reciente evidencia de que estos bosques no garantizarían completamente un balance estable de carbono entre la atmósfera y biósfera a largo plazo (Brienen et al. 2015). Estos escenarios negativos deben revertirse a través de acciones integrales, rápidas y factibles para reducir las emisiones por deforestación y degradación forestal, además de actividades integrales para la conservación, manejo sostenible e incremento de las reservas de carbono (REDD+) en el marco de los acuerdos de la Convención Marco de las Naciones Unidas sobre Cambio Climático (Jagger et al. 2013).

Así mismo, es bien conocido que la Amazonía es una de las regiones biogeográficas que presenta una alta diversidad biológica en comparación a otros bosques tropicales en diferentes latitudes del mundo (Strassburg et al. 2010), y que a pesar de las tasas de deforestación, así como el incremento reciente de las tasas de mortalidad de árboles (Bennett et al. 2015, Brienen et al. 2015), continúa presentando un alto potencial para el almacenamiento de carbono como uno de los servicios ecosistémicos de mayor impacto global, con aproximadamente $93 \mathrm{Pg}$ de carbono entre la biomasa aérea y subterránea (Malhi et al. 2006). Estos procesos tienen importante implicaciones en el contexto REDD+ que tiene como objetivo principal fomentar trayectorias de desarrollo de baja emisión, aumentando el valor de los bosques en relación a diferentes tipos de uso de la tierra (Angelsen y McNeill 2013). Por este motivo, el acuerdo de Cancún de 2010 estableció un conjunto de salvaguardias sociales y ambientales para la implementación de las actividades de REDD +, que promuevan la conservación de los bosques naturales y sus servicios ecosistémicos, además de proporcionar otros beneficios socioambientales (Jagger et al. 2013). En este sentido, se destaca que para mantener los objetivos de REDD + se requiere la transformación de las actividades económicas dentro y fuera de los bosques, que son reconocidos como las causas directas e indirectas de la deforestación y la degradación forestal (Angelsen y McNeill 2013, Jagger et al. 2013).

El Amazonas es reconocida como la cuenca de mayor ocupación indígena en el trópico (Roosevelt 2013), con más de 350 pueblos indígenas que han venido dependiendo de estos bosques desde hace más de 13.000 años como fuente de bienes y servicios, y que según evidencias arqueológicas, han sido obtenidos principalmente a través de la agricultura de tala y quema a pequeña escala, la recolección de alimentos y la cacería (Arroyo-Kalin 2012, Bush et al. 2015, Piperno et al. 2015). Esta relación del hombre con la naturaleza es ampliamente reconocida como un sistema socioecológico donde los ecosistemas son afectados para el aprovechamiento de recursos que favorecen el bienestar humano (Cumming y Allen 2017). En gran parte del Amazonas no existían asentamientos humanos permanentes con sistemas agrícolas estables, lo que habría permitido la regeneración natural del bosque a largo plazo (Bush et al. 2015). La alta biodiversidad de estos ecosistemas ha sido fundamental para la supervivencia de estas comunidades indígenas hasta la actualidad (Arroyo-Kalin 2012), manteniendo el mismo patrón ancestral de agricultura de subsistencia (Piperno et al. 2015). Por este motivo, actualmente existe una mayor preocupación por el incremento acelerado de poblaciones sedentarias con una fuerte influencia de la cultura occidental dentro de la Amazonía y de la inmigración de población mestiza que provoca la intensificación en el uso de la tierra a través de sistemas agrícolas itinerantes, así como el aprovechamiento de recursos forestales con fines económicos. Sin embargo, no existen publicaciones sobre la relación de estos tipos de uso de la tierra con estimaciones de emisión de carbono para toda la cuenca, sólo se reconoce que contribuyen significativamente con la deforestación y degradación forestal (Lapola et al. 2013, Aragão et al. 2014).

Más recientemente, las reformas agrarias en algunos países de la cuenca, basadas en medidas de desarrollo económico a partir del año 1960, incentivaron procesos de colonización con la expansión de la frontera agrícola de forma heterogénea, intensificándose el proceso de deforestación a partir del año 1970, principalmente entre el sur y sureste de la cuenca con el establecimiento de plantaciones de soja, ganadería y tala selectiva (Nepstad et al. 2014, Rodrigues-Filho et al. 2015, Aguiar et al. 2016). En este contexto, una primera aproximación para alcanzar los objetivos REDD+, es comprender los patrones y procesos asociados con los cambios en el uso de la tierra, a través del análisis de diferentes causas antropogénicas y biofísicas de la deforestación y degradación forestal (figura 1). Estas causas también son reconocidas como predictores durante el uso de modelos matemáticos, especialmente cuando se analiza su relación con variables respuestas (cobertura vegetal, biomasa, riqueza) asociadas con la deforestación y degradación (Laurance et al. 2002, D’Oliveira et al. 2011, Wandelli y Fearnside 2015). Los predictores antropogénicos permiten estimar los impactos causados 
por el hombre durante la explotación del bosque para la provisión de bienes y servicios ecosistémicos (Perz y Skole 2003, Lapola et al. 2013). Por otro lado, los predictores biofísicos proporcionan información sobre el efecto de la variabilidad ambiental (clima, geoformas, suelos) en la dinámica de los ecosistemas forestales (Laurance et al. 2002). En todo caso, estos predictores están actuando sinérgicamente (Laurance et al. 2002, Aragão et al. 2014, Rodrigues-Filho et al. 2015). Si bien, existen algunas investigaciones que explican cómo diferentes predictores antropogénicos y biofísicos influyen directamente en la dinámica de deforestación de la Amazonía (Laurance et al. 2002, Verburg et al. 2014, Pérez et al. 2015 RodriguesFilho et al. 2015, Aguiar et al. 2016), todavía es necesario entender como los procesos sociales y económicos también pueden ser importantes causas de deforestación que deben considerarse para el futuro de la conservación de los bosques en la región.

Por otra parte, todavía es necesario seguir evaluando los impactos causados por los cambios en el uso de las tierras forestales sobre la biodiversidad y carbono bajo un mismo escenario, sobre todo evaluando la relevancia de actividades REDD+ (Thompson et al. 2012, Kapos et al. 2012, Villa et al. 2015). De esta forma, ha sido propuesta la implementación integral de actividades REDD+, como alternativas para la conservación, recuperación e incremento de carbono y biodiversidad en la región, a través de sistemas agrícolas sustentables, restauración simultánea de áreas forestales degradadas y rehabilitación de áreas a través de la agroforestería (Kapos et al. 2012, Villa et al. 2015). No obstante, dentro de las políticas de desarrollo rural y resultados de investigaciones científicas, existen pocas propuestas sistemáticas sobre los cambios en uso de la tierra dentro del sistema socioecológico, que además contemple diferentes estrategias REDD+ bajo una perspectiva de biodiversidad y carbono simultáneamente (figura 1).

Con el propósito de contribuir con la eficiencia en el manejo y conservación de bosques en la Amazonía que se encuentran bajo modificación humana, en este artículo se desarrolla una revisión descriptiva y analítica para contextualizar las características de predictores biofísicos y antropogénicos de deforestación y degradación, que deberían considerarse como premisas para la integración de activi-
Comunidad vegetal

(Variables respuesta)
Función ecosistémica (Almacenamiento de carbono)

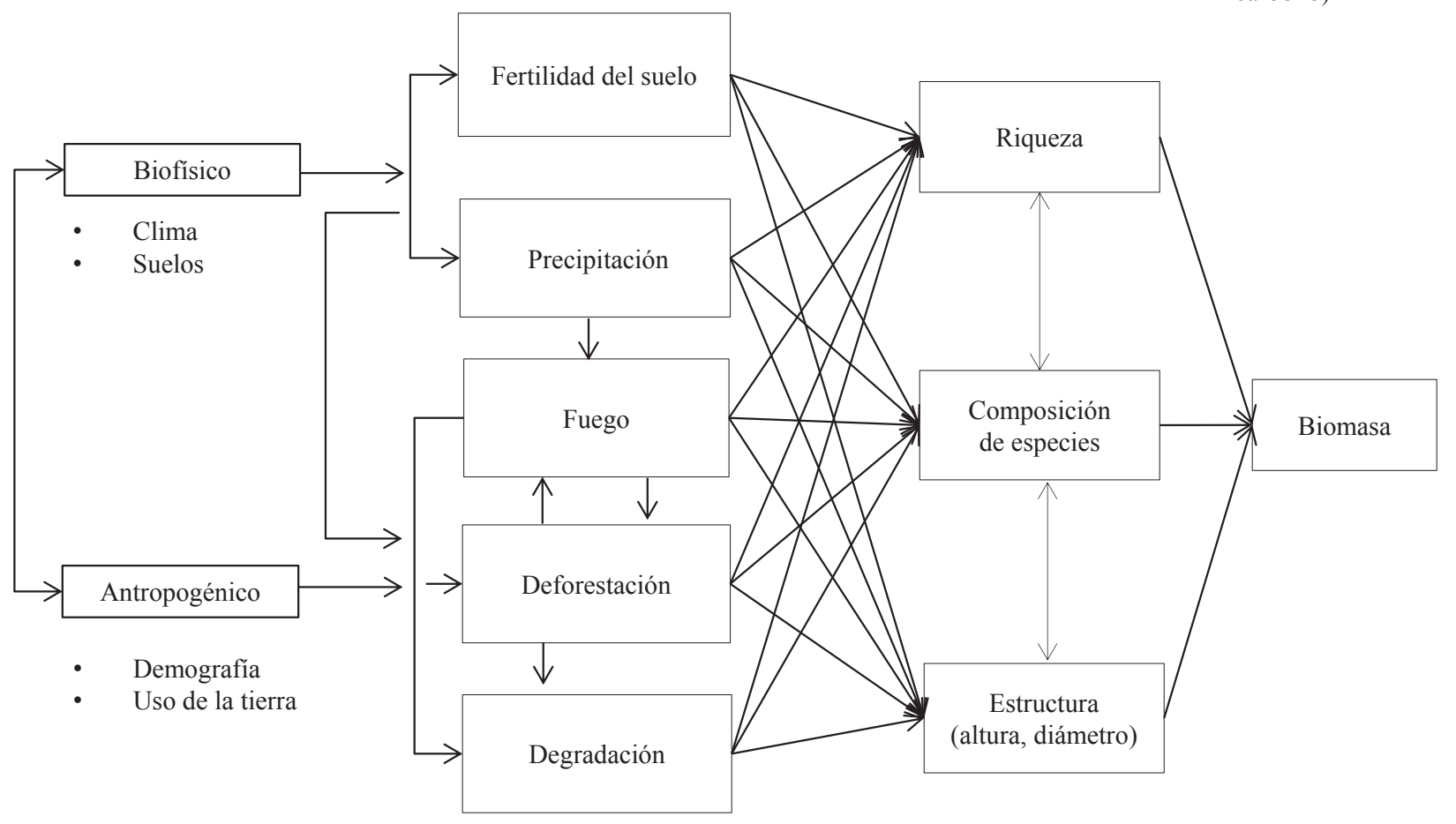

Figura 1. Diagrama conceptual indicando como los predictores biofísicos y antropogénicos afectan la estructura de las comunidades vegetales, y como los predictores y la riqueza (número de espécies), composición florística y estructura (altura, diámetro, densidad de la madera) de las comunidades afectan simultáneamente el almacenamiento de carbono en la biomasa.

Conceptual diagram indicating how biophysical and anthropogenic predictors affect the plant communities' structure, and how the predictors and plant communities' structure affect simultaneously carbon storage in biomass. 
dades REDD+. Por lo tanto, se proponen como objetivos, 1) describir la relación biodiversidad-función ecosistémica haciendo énfasis en la importancia del almacenamiento de carbono como servicio ecosistémico; 2) analizar los posibles impactos sinérgicos de predictores antropogénicos y biofísicos de la deforestación. Finalmente, 3) discutir actividades REDD+ ajustadas a la dinámica del sistema socioecológico, con énfasis en la conservación, recuperación, e incremento del carbono y biodiversidad de la Amazonía. Las actividades REDD+ consideradas para el desarrollo de esta revisión han sido seleccionadas de protocolos actuales de la Unión Internacional de Organizaciones de Investigación Forestal (International Union of Forest Research Organizations, IUFRO), para el entendimiento de la relación entre "biodiversidad, carbono, bosques y personas" como componentes clave para el desarrollo de propuestas REDD+ (Kapos et al. 2012).

\section{RELACIÓN BIODIVERSIDAD-FUNCIÓN ECOSISTÉMICA}

El análisis de la relación biodiversidad y función ecosistémica (BEF, por sus siglas en inglés) ha sido muy importante para entender como la riqueza de especies afecta diferentes procesos ecológicos de los ecosistemas (Oliver et al. 2015), así como por la necesidad práctica de conservar y manejar servicios ecosistémicos que tienen transcendentales impactos sobre la humanidad (Cardinale et al. 2012). Sin embargo, aunque es evidente la disminución de la biodiversidad a nivel mundial (Strassburg et al. 2010), no se conoce cuál es el impacto sobre la pérdida de funciones ecosistémicas (Oliver et al. 2015), y menos sobre las tendencias futuras de demanda de bienes y servicios a escala regional. La mayoría de los estudios sobre mecanismos BEF se han enfocado principalmente en la productividad de biomasa vegetal, debido a que el carbono acumulado en la vegetación es determinante en el balance global con la atmósfera (Lasky et al. 2014, Brienen et al. 2015). Por este motivo, comprender el papel de la biodiversidad en el mantenimiento de las funciones ecosistémicas bajo diferentes niveles de degradación generados por un complejo sistema socioecológico en Amazonas (figura 1), es fundamental para establecer medidas de conservación y estrategias efectivas REDD+. De igual modo, es necesario identificar la importancia relativa de los predictores de deforestación y de gradación, estimando los impactos sobre las reservas de carbono en la biomasa (figura 2A) através de gradientes climáticos de la Amazonía (figura 2B), así como en diferentes tipos de bosques con diferentes etapas de regeneración natural (figura $2 \mathrm{C}$ ).

Se ha demostrado como los rasgos funcionales de especies arbóreas pueden ser buenos predictores de la producción de biomasa durante diferentes etapas de sucesión secundaria (Lasky et al. 2014), sobre la dinámica de la relación biodiversidad-carbono que es dependiente de la escala espacial (Pooter et al. 2015), incluso en sistemas agroforestales (Ali y Mattsson 2017). Sin embargo, es poco lo que se conoce sobre el efecto que tienen las especies más dominantes en estos procesos ecosistémicos. Por ejemplo, a escala de toda la Amazonía, se ha estimado que apenas $1 \%$ del total de especies de árboles son responsables del $50 \%$ de la productividad y almacenamiento de carbono en toda la cuenca, distribuidos en aproximadamente 182 especies hiperdominantes de biomasa, y 184 especies hiperdominantes de productividad (Fauset et al. 2015). Esta premisa es fundamental para la selección de especies durante la restauración de servicios ecosistémicos con actividades REDD + , conectando la importancia relativa de los rasgos funcionales a escala de individuos hasta escala de ecosistemas, con la finalidad de categorizar áreas potenciales de restauración en función de filtros ambientales y demanda del sistema socioecológico (figura 2D). Al mismo tiempo, la información sobre procesos biofísicos podría ser utilizada para generar indicadores de las funciones ecosistémicas, bien para saber el nivel de vulnerabilidad de los ecosistemas y gestión de riesgo frente a presiones de cambios climáticos y antropogénicos, o para evaluar y predecir la capacidad de regeneración de los ecosistemas a través de acciones REDD+.

Cabe destacar que la composición de especies de las comunidades vegetales está cambiando rápidamente a causa de los cambios en el uso de la tierra y el cambio climático, con consecuencias potencialmente graves para la capacidad de recuperación de funciones de los ecosistemas (Cardinale et al. 2012, Oliver et al. 2015). Una de las principales consecuencias es que la tasa de mortalidad de árboles a nivel mundial está incrementando a causa de intensos y prolongados periodos de sequía, incluyendo el Amazonas, donde árboles grandes están presentando las mayores tasas en comparación con árboles pequeños (Bennett et al. 2015). Estos resultados podrían cuestionar la importancia relativa de las especies hiperdominantes en el secuestro y almacenamiento de carbono en Amazonas, porque también podría incrementar la tasa de mortalidad de las especies funcionalmente mas importantes. Finalmente, se resalta la relevancia del fuego en la dinámica de la biomasa y carbono en bosques de la Amazonía, que representan una constante amenaza de degradación (Aragão et al. 2014), principalmente en bosques estacionales semideciduales, sabanas estacionales y bosques mixtos con dominancia de bambú, durante periodos de prolongadas sequías interanuales (Brando et al. 2014, Nogueira et al. 2015, Aguiar et al. 2016).

\section{PREDICTORES BIOFÍSICOS}

Ha sido bien demostrado que los suelos de la Amazonía son altamente lixiviados, predominantemente ácidos y de baja fertilidad, mientras que la alta asignación de biomasa está influenciada principalmente por un ciclo cerrado de nutrientes que depende de la alta producción de hojarasca, altas tasas de descomposición, y alta eficiencia en el uso 
de nutrientes (Quesada et al. 2012). Sin embargo, todavía es poco conocida la relación existente de estos procesos ecosistémicos con la biodiversidad y sobre todo, su repercusión en el funcionamiento de los ecosistemas de acuerdo a los predictores biofísicos de cada localidad.

Poorter et al. (2016) demostraron que los bosques secundarios neotropicales, incluyendo los de la Amazonía, difieren drásticamente en su capacidad de recuperación de biomasa (promedio de 66 años para recuperar $90 \%$ de la biomasa aérea), principalmente impulsado por la variación en la disponibilidad de agua, debido a que las altas precipitaciones y el bajo déficit hídrico, permiten extender la temporada de crecimiento e incrementar la producción de biomasa. Así mismo, explican que la fertilidad de suelos tiene un efecto positivo significativo en la recuperación relativa de biomasa, pero ningún efecto sobre la recuperación absoluta debido a que las grandes diferencias con el macroclima pueden anular efectos más sutiles de la fertilidad del suelo. Por esto mismo, analizando los patrones y procesos determinantes en la estabilidad de la estructura
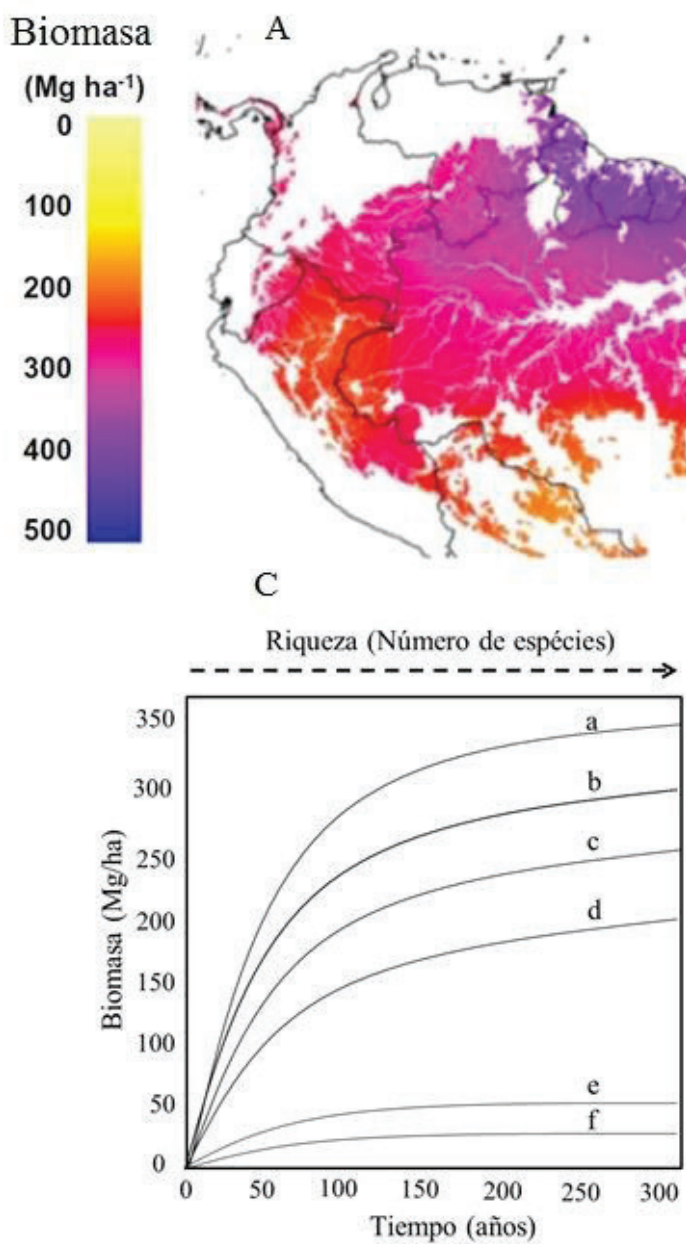

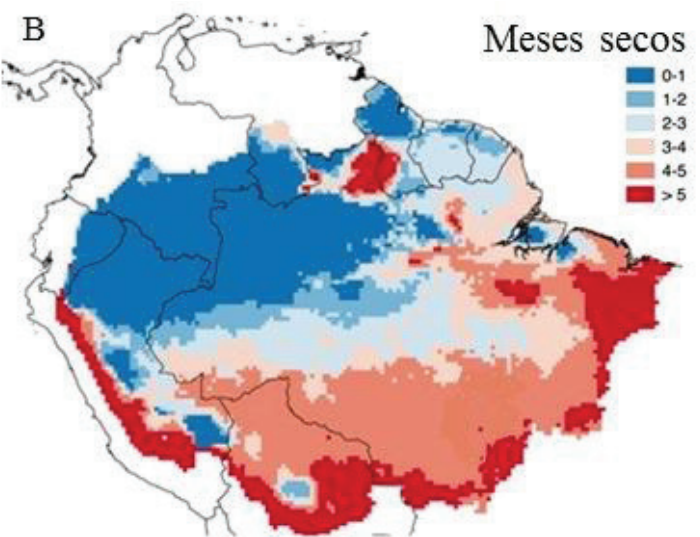

D

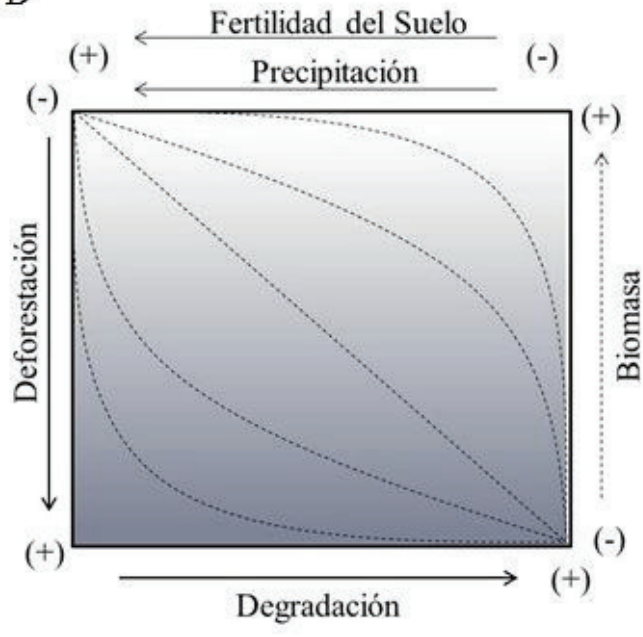

Figura 2. Mapa de distribución de biomasa sobre el suelo en la cuenca Amazónica (2A), adaptado de Mitchard et al. (2014). Mapa de distribución del tiempo promedio de duración de la estación seca (número de meses con precipitación $\leq 100 \mathrm{~mm}$ ) en la cuenca Amazónica (2B), adaptado de Brando et al. (2014). Tendencias estimadas de acumulación de biomasa en bosques de la Amazonía, bosque lluvioso denso de tierras bajas no inundable (a), bosque lluvioso denso de tierras bajas inundable (b), bosque lluvioso estacional semidecidual no inundable (c) bosque lluvioso estacional semidecidual inundable (d), sabana boscosa estacional (e), sabana arbolada estacional (f), adaptado de Nogueira et al. (2015). Se indica tendencia teórica de riqueza en función del tiempo (2C). Diagrama conceptual indicando tendencias teóricas de como predictores biofísicos (Precipitación y fertilidad del suelo) y deforestación influyen en la pérdida de biomasa y degradación forestal (2D).

Above-ground biomass (AGB) maps of Amazon basin (2A), adapted from Mitchard et al. (2014). Average dry season length (number of months with precipitation $\leq 100 \mathrm{~mm}$ ) maps of Amazon basin (2B) adapted from Brando et al. (2014). Estimated pattern of biomass and carbon accumulation in Amazonian forests: dense rainforest on nonflooding lowlands (A), dense rainforest on flooded lowland (B), semideciduous seasonal nonflooding forest (C), semideciduous seasonal flooding forest (D), seasonal forest savanna (E), and seasonal woodland savanna (F). Adapted from Nogueira et al. (2015). Theoretical richness as a function of time is indicated (2C). Conceptual diagram indicating theoretical trends as biophysical predictors (Precipitation and soil fertility) and deforestation induced biomass loss and forest degradation (2D). 
y funcionamiento de los bosques amazónicos (maduros y secundarios) a través de gradientes climáticos, principalmente por el efecto de la precipitación y suelos como predictores biofísicos, es posible estimar la contribución relativa de los efectos que pueden generar los cambios en el uso de la tierra (figura 1). Es decir, que las futuras investigaciones sobre BEF y acciones REDD+ en la Amazonía, también deberían enfocarse en el análisis simultáneos de predictores antropogénicos y biofísicos sobre variables respuestas como la recuperación de biomasa (figura 2D).

Una primera aproximación sobre algunos predictores biofísicos que influyen en la deforestación de algunas regiones de la Amazonía, ha sido analizando el efecto que tienen los menores valores de precipitación, mayor déficit hídrico (> 5 meses secos, figura 2B), y suelos con baja fertilidad, sobre la vulnerabilidad y capacidad de regeneración de los bosques lluviosos estacionales semideciduales y sabanas estacionales en el sureste de la cuenca (Brando et al. 2014, Nogueira el at. 2015), siendo una región conocida como el "arco de deforestación" (Laurance et al. 2001). La mayor concentración de población rural, y la continua incidencia de fuegos antropogénicos en esta subregión, son predictores importantes de la degradación forestal. Además, actualmente continúan siendo las zonas con mayor deforestación y fragmentación de toda la cuenca (Brando et al. 2014, Lapola et al. 2014, RodriguesFilho et al. 2015, Aguiar et al. 2016). Se presume que la tendencia actual de diferentes predictores antropogénicos anteriormente discutidos, y en consecuencia mayores tasas de deforestación para la expansión de la frontera agrícola en esa región del Amazonas, esta gestando un círculo vicioso que está trascendiendo a un irreversible "arco de degradación".

Es bien conocido que el fuego ha sido utilizado durante muchas generaciones como un medio para el manejo de la tierra con fines agrícolas en la Amazonía (ArroyoKalin 2012, Roosevelt 2013, Bush et al. 2015, Piperno et al. 2015), sin embargo, son limitados los estudios que demuestren el efecto sobre las propiedades biológicas, físicas, químicas del suelo, y, en consecuencia, sobre las trayectorias de sucesión secundaria de los bosques en Amazonas, principalmente evaluando la capacidad de recuperación de biomasa y biodiversidad (D'Oliveira et al. 2011). En estudios recientes, se concluye que los distintos regímenes de severidad y frecuencia del fuego como práctica agrícola, provocan marcadas diferencias en la regeneración natural de bosques amazónicos, tanto en su composición de especies como en la acumulación de biomasa (D'Oliveira et al. 2011, Wandelli y Fearnside 2015). Bajo este escenario, Aragão et al. (2014) destacan la importancia de investigar el efecto multifactorial de predictores antropogénicos y biofísicos (por ejemplo fuego y sequía) sobre la deforestación y emisión de carbono, así como sobre la capacidad de recuperación de funciones ecosistémicas (figura 3). Sin embargo, existe una primera evidencia sobre aumentos abruptos en la mortalidad de árboles (226 \%) in- ducidos por incendios anuales durante un evento de sequía en el Suerte de la Amazonía (Brando et al. 2014). Por otro lado, estos investigadores observaron como consecuencia una marcada disminución de la cobertura del dosel (23\%) y biomasa viva sobre el suelo (12\%), favoreciendo la invasión generalizada por gramíneas inflamables a través del área de borde forestal $(80 \%)$ donde los incendios fueron más intensos.

\section{PREDICTORES ANTROPOGÉNICOS}

Existen varios estudios desarrollados en algunas áreas de la Amazonía que describen algunos factores que influyen en la deforestación y degradación forestal, basados en criterios ambientales, culturales, sociales, económicos y políticas de desarrollo rural (Laurance 2002, Peres et al. 2010, Arroyo-Kalin 2012, Espindola et al. 2012, Diniz et al. 2013, Nepstad et al. 2014, Verburg et al. 2014, Piperno et al. 2015). No obstante, continúa siendo un desafío lograr integrar sus relaciones después de diferentes escenarios de modificación humana a escala de toda la cuenca. La mayoría de estos cambios en el uso de la tierra son el resultado de una combinación de predictores antropogénicos, sin embargo, para entender esta compleja dinámica a escala regional, también es fundamental profundizar en la evaluación de procesos sociodemográficos que actúan a escala local. En este sentido, Godar et al. (2014) observaron que casi la mitad $\left(36.158 \mathrm{~km}^{2}\right)$ de la deforestación en la Amazonía brasileira entre 2004 y 2011 ocurrieron en áreas de productores con propiedades más grandes $(>500$ ha), mientras que sólo el $12 \%\left(9.720 \mathrm{~km}^{2}\right)$ ocurrieron en áreas dominadas por propiedades de pequeños productores $(<100$ ha). Además, los bosques en áreas dominadas por pequeños agricultores fueron menos fragmentadas y degradadas. Sin embargo, aunque las tasas anuales de deforestación disminuyeron durante este período en 68-85 $\%$ para todos los actores, la contribución de los mayores terratenientes $(>2.500 \mathrm{ha})$ en la deforestación anual disminuyó a lo largo del tiempo (63\% entre 2005 y 2011), mientras que la de los pequeños productores aumentó 69 $\%$ durante el mismo período. Ahora el mayor desafío es lograr determinar estos patrones de uso de la tierra en los demás países que hacen parte de la cuenca.

Por otro lado, Espindola et al. (2012) encontraron notables diferencias en la dinámica de uso de tierras agrícolas en dos años diferentes (1997 y 2007) entre los estados Rondônia, Mato Grosso y Pará, basados en modelos de i) estructura de asentamientos urbanos y vialidad, así como de ii) desarrollo agrario. Estos investigadores concluyen que la distancia a la carretera y el número de nuevas familias asentadas, tuvo mayor impacto en la deforestación y dinámica en el uso de la tierra durante el 2007, lo que indica que esta relación podría ser más fuerte con el transcurso del tiempo. Así mismo, Lapola et al. (2013) analizan la ocupación de la Amazonía brasileña cuantificando las relaciones espaciales y temporales de la deforestación y 
ocupación de la tierra, argumentado que la aplicación de políticas de distribución y tenencia de la tierra debe transcender a la autorregulación del mercado para amortiguar los efectos detrimentales de la intensificación agrícola que privilegia la optimización en el uso de la tierra. Del mismo modo, Pérez y Skole (2003) probando modelos matemáticos de distribución de bosques secundarios en la cuenca del Amazonas, usando variables sociales explicativas como los asentamientos humanos sedentarios y las actividades tradicionales para evaluar el efecto sobre la deforestación, encontraron una relación positiva y significativa con las variaciones espaciales entre diferentes subregiones. Sin embargo, en este mismo estudio los nuevos asentamientos y actividades no tradicionales (cultivos anuales y pastizales) tuvieron una fuerte relación en la mayoría de los modelos analizados. La investigación de Perez y Skole (2003) representa un trabajo pionero de referencia regional que demuestra como los predictores antropogénicos son fundamentales para entender el proceso de deforestación en Amazonas, y por lo tanto, conocer la magnitud de las acciones humanas en la demanda de recursos forestales como premisas básicas para la definición de actividades REDD+.
Las altas tasas de inmigración y de crecimiento poblacional han sido considerados predictores antropogénicos determinantes en los cambios en el uso de la tierra de la Amazonía (Laurance et al. 2002), principalmente a través de la agricultura, encontrándose que la población mestiza brasileña aumentó aproximadamente de 2 a 20 millones de personas desde el año 1960, debido a la inmigración desde otras regiones de Brasil, y en consecuencia incrementado las áreas agrícolas en esta región (Laurance et al. 2001, Diniz et al. 2013, Lapola et al. 2013). No obstante, la ampliación de las redes viales y pavimentación de las carreteras que ha favorecido la colonización de nuevas áreas, es una de las principales variables que mejor explica cómo la deforestación se está expandiendo paulatinamente hacia la región central de la Amazonía (Verburg et al. 2014). De igual modo, se resalta que la pavimentación de carreteras que cruzan el bioma amazónico está permitiendo un mayor flujo de transporte de productos agroforestales a los mercados europeos y asiáticos, así como también proporcionando un mejor acceso para los productores locales de soja (Nepstad et al. 2014, Verburg et al. 2014, Rodrigues-Filho et al. 2015), lo que esta promoviendo simultáneamente un

$(-)$
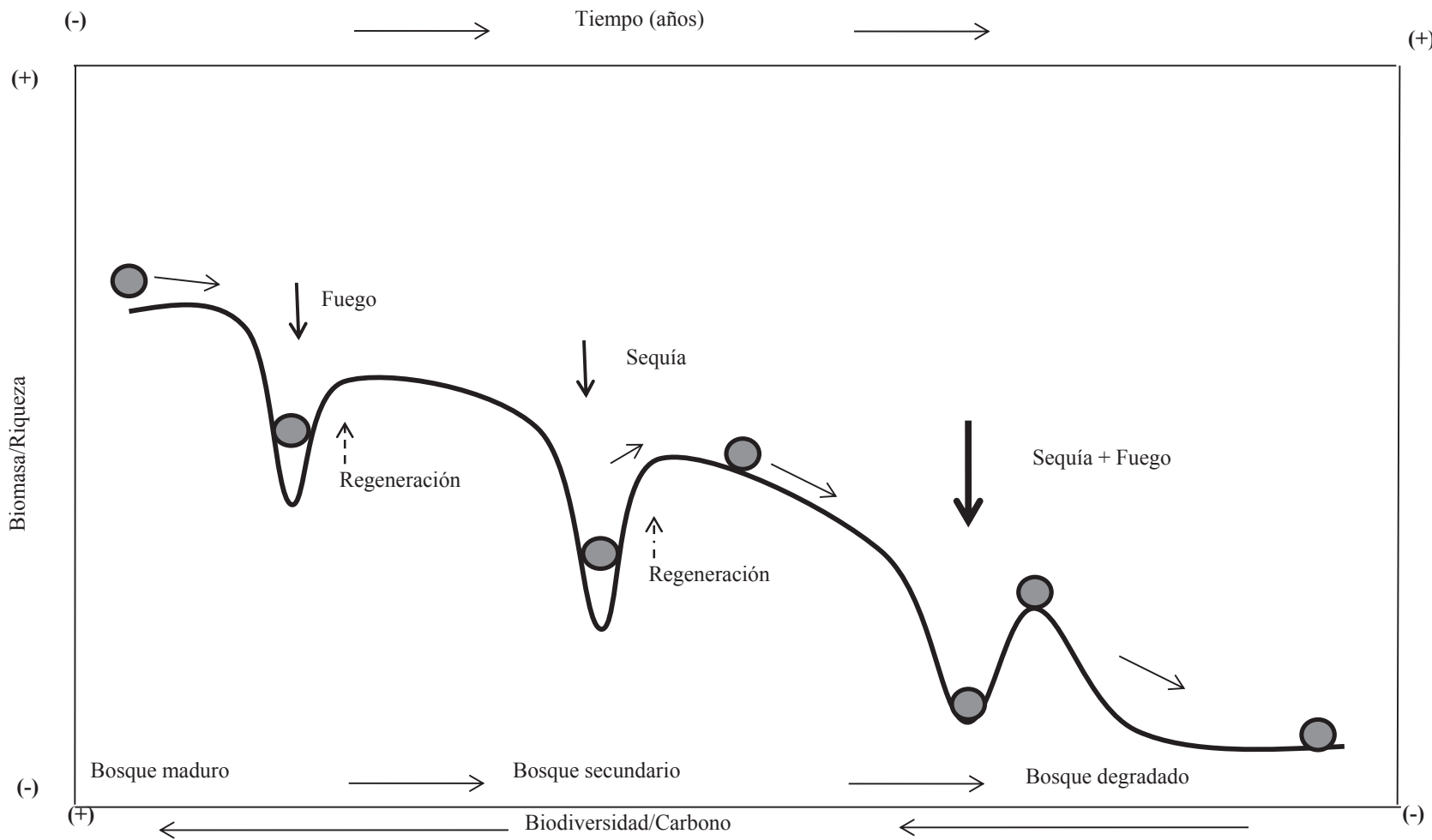

Figura 3. Representación clásica del modelo de resiliencia de los ecosistemas. Las bolas representan los estados de un ecosistema (donde un estado se define por un conjunto de variables como la biomasa y riqueza de especies), las flechas continuas representan las perturbaciones (fuego, sequía, o sequía y fuego), los valles representan la pérdida de biomasa o riqueza, y los picos representan umbrales (recuperación de biomasa o riqueza, flechas discontinuas). Adaptado de Oliver et al. (2015).

Classical model representations of ecosystem resilience. Balls represent states of an ecosystem (where a state is defined by a set of variables such as biomass and species richness), continuous arrows represent disturbances (fire, drought, or drought-fire), valleys represent biomass or richness loss, and peaks represent thresholds (biomass or richness recovery, dashed arrows). Adapted from Oliver et al. (2015). 
aumento de la inmigración hacia las mismas áreas con mayor deforestación.

La dinámica demográfica depende en gran medida de la inestabilidad de las poblaciones por las demandas sociales y económicas (fuentes de trabajo y medios de vida), ya que la economía rural de la región se basa en gran medida en los sectores de explotación (forestales, agrícolas), a partir de los recursos de la biodiversidad (Aguiar et al. 2013) y acceso a los mercados (Lapola et al. 2013, Verburg et al. 2014). En cualquier caso, se propone hacer una relación directa de los predictores antropogénicos de deforestación, como el efecto de sistemas viales y dinámica de poblaciones sobre la demanda y manejo de bosques para la provisión de bienes y servicios ecosistémicos. Hasta ahora, solo Lapola et al. (2013) y Aguiar et al. (2016) incursionaron en el análisis integral de procesos sociodemográficos y socioeconómicos de la deforestación, sobre la emisión de carbono a la atmósfera en el Amazonas brasileño.

\section{INTEGRANDO ACTIVIDADES REDD+}

Hasta ahora es posible identificar, en forma general, los impactos que pueden tener los predictores antropogénicos y biofísicos de deforestación; pero es evidente que todavía falta mucho por investigar sobre la transcendencia e impactos cuantitativos que pueden tener sobre las actividades
REDD+ para recuperar y conservar la relación carbonobiodiversidad. Actualmente son reconocidas dos principales estrategias para mitigar el incremento de carbono en la atmósfera a causa de la deforestación, i) controlando la reducción de emisiones a través de la conservación y manejo sustentable de los ecosistemas forestales, e ii) incrementando la producción de biomasa vegetal como importantes reservas de carbono en la biosfera, a través de prácticas de restauración forestal y rehabilitación de áreas forestales degradadas como parte de las actividades integrales REDD+ (figura 5). En estas estrategias está implícito un impacto positivo sobre la biodiversidad, aunque resultaría más conveniente enfatizar la importancia del balance biodiversidad-función ecosistémica en términos REDD+.

Generalmente los estudios de sucesión forestal en Amazonas se han desarrollado en áreas agrícolas que fueron destinadas para un único ciclo de cultivo. Después del abandono del sistema agrícola, estas áreas son tradicionalmente conocidas como barbechos (tiempo de descanso de la tierra), porque posiblemente podrían ser usadas para nuevos ciclos de cultivos en años posteriores (figura 4). Sin embargo, se ha demostrado que el uso sucesivo de las mismas áreas agrícolas abandonadas que se encuentran con diferentes tiempos de regeneración, por dos o más ciclos agrícolas adicionales, influye considerablemente en la pérdida de la biodiversidad y biomasa en la Amazo-

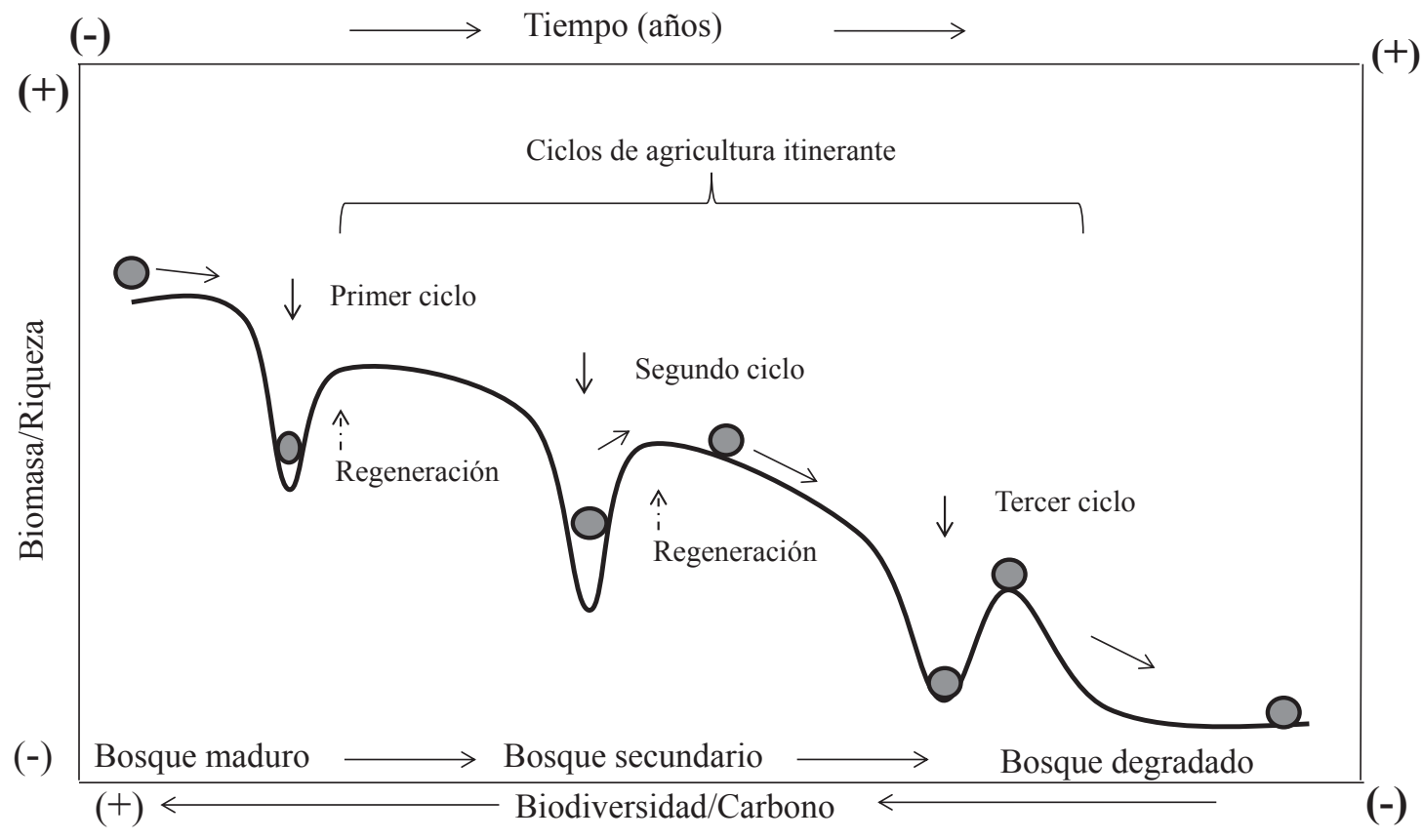

Figura 4. Representación clásica del modelo de resiliencia de los ecosistemas. Las bolas representan los estados de un ecosistema (donde un estado se define por un conjunto de variables como la biomasa y riqueza de especies), las flechas continuas representan las perturbaciones (tala para sistemas agrícolas itinerantes), los valles representan la pérdida de biomasa o riqueza y los picos representan umbrales (recuperación de biomasa o riqueza, flechas discontinuas). Adaptado de Oliver et al. (2015).

Classical model representations of ecosystem resilience. Balls represent states of an ecosystem (where a state is defined by a set of variables such as biomass and species richness), continuous arrows represent disturbances (clear-cutting for shifting cultivation), valleys represent biomass or richness loss, and peaks represent thresholds (biomass or richness recovery, dashed arrows). Adapted from Oliver et al. (2015). 
nía (Thompson et al. 2012, Aragão et al. 2014, Villa et al. 2015, Wandelli y Fearnside 2015, Aguiar et al. 2016). Esta perspectiva comienza a tener mayor preocupación por el incremento de las poblaciones sedentarias, que dependen de los bosques secundarios próximos para el uso sucesivo y ampliación de las áreas agrícolas. En este contexto, se empieza a concebir una agricultura basada en la biodiversidad y carbono, especialmente a través de la agroforestería que ya presenta importantes experiencias con parcelas permanentes en varias subregiones de la cuenca (Porro et al. 2012). Cabe indicar, que varios de los sistemas agroforestales (SAF) más antiguos de la cuenca presentan árboles maduros con una disminución de su capacidad productiva, por lo que se resalta la importancia de investigar el impacto de los SAF con manejo de ciclos sucesivos, especialmente en áreas permanentes para evitar la deforestación de nuevas áreas forestales (figura 6).

Según Brienen et al. (2015) los bosques del Amazonas después de haber sido importantes sumideros de carbono durante los últimos 30 años, podrían perder su capacidad potencial de acumulación de carbono a largo plazo como se esperaba inicialmente, comenzando a ser fuentes de carbono a la atmósfera por el aumento de la tasa de crecimiento y mortalidad de los árboles, contribuyendo en un balance negativo de carbono entre la biosfera y atmósfera. Este resultado indica que si los bosques maduros no garantizan un

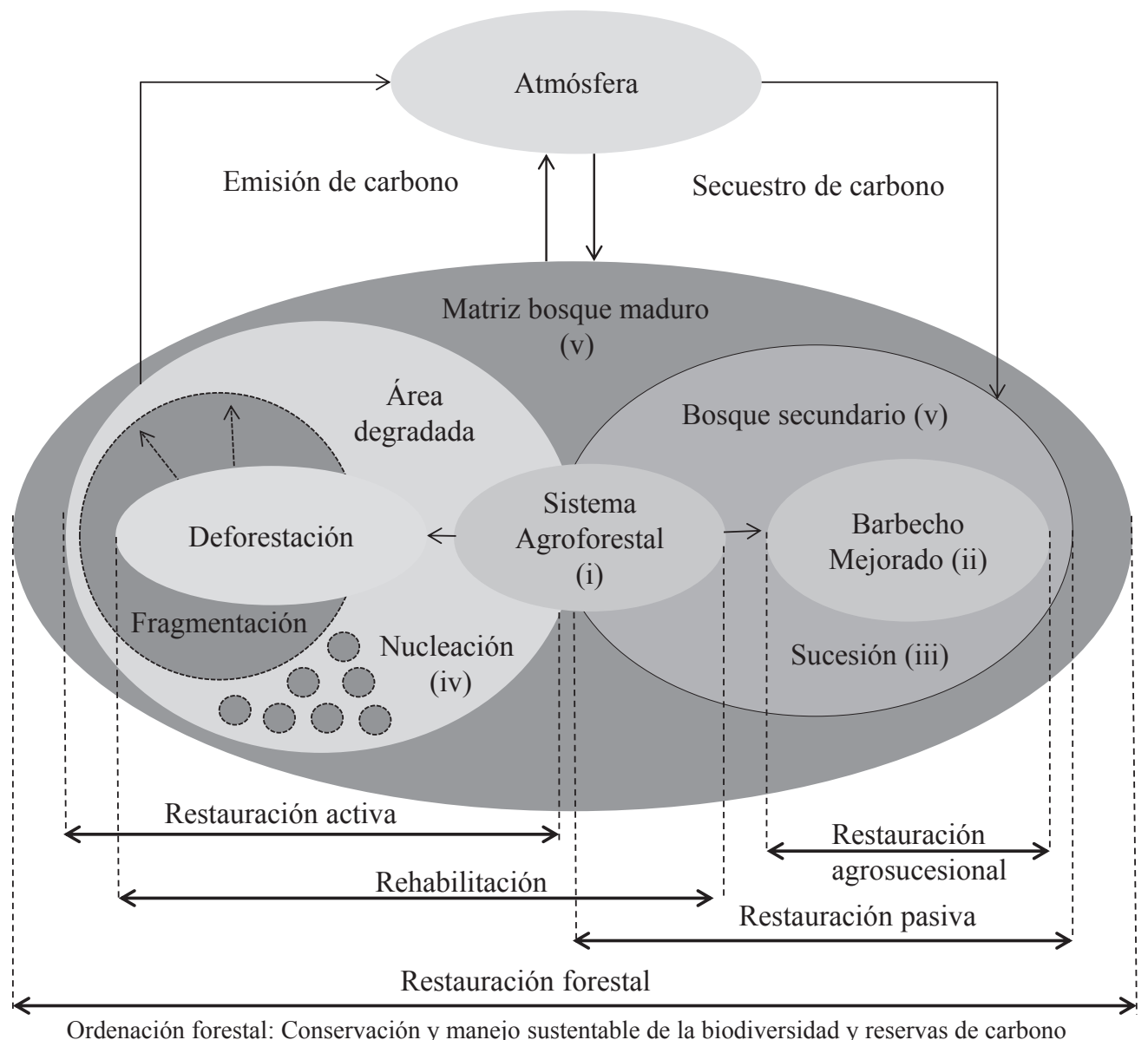

Figura 5. Integración de diferentes tipos de manejo de bosques con impactos en las acciones REDD+, como la reducción de las emisiones de carbono por deforestación, y degradación forestal, a través de la conservación de bosques primarios, manejo sustentable de bosques secundarios, e incremento de las reservas de carbono y biodiversidad. Se proponen las siguientes medidas de rehabilitación y restauración: i) sistemas agroforestales, ii) barbecho mejorado como sistema agrosucesional, iii) sucesión secundaria como mecanismo de restauración pasiva, iv) nucleación como restauración activa, v) conservación de bosques maduros y secundarios. Adaptado de Villa et al. (2015).

Integration of different types of forest management with impacts on REDD+ actions such as the reduction of carbon emissions from deforestation and from forest degradation, through conservation of old forests, sustainable management of second-growth forests, and increase in carbon stocks and biodiversity. The following rehabilitation and restoration alternatives are proposed: agroforestry systems as a rehabilitation method (i), improved fallow as an agrosucesional system (ii), succession as a passive restoration mechanism (iii), and nucleation as active restoration (iv), old growth and second-growth forest conservation (v). Adapted from Villa et al. (2015). 


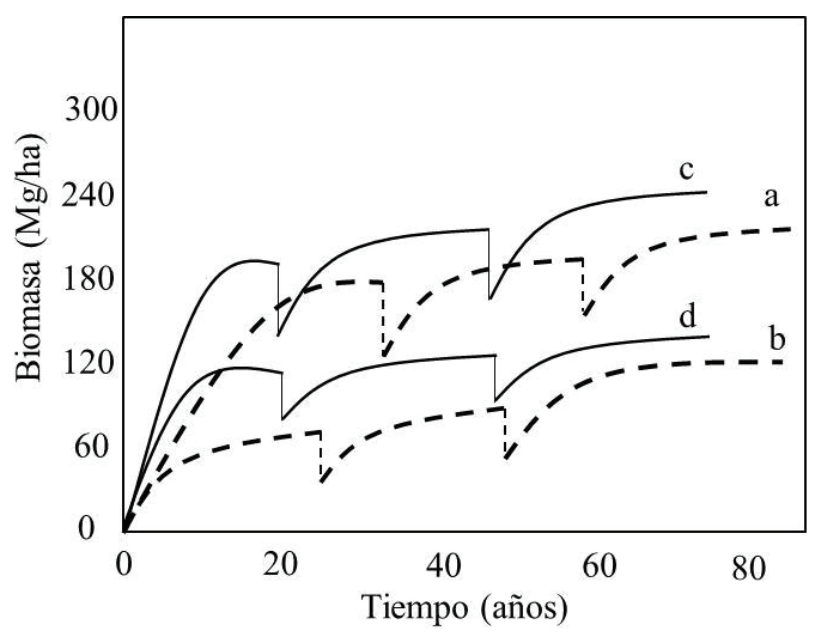

Figura 6. Acumulación potencial de biomasa y carbono bajo diferentes tipos de manejo de bosques con impactos en las acciones REDD + , principalmente en sistemas agroforestales multiestratos dominado por cacao (líneas de trazos) con cosechas sucesivas cada 30 años (a), multiestratos con especies frutales, maderables, y cacao, con cosechas cada 25 años (b). Se indican tendencias comparativas para plantaciones forestales más comunes en el trópico y Amazonas (líneas continuas), como Swietenia macrophylla King. (c), y Tectona grandis L.f. (d), con cosechas aproximadamente cada 20 años para cada especie (Adaptado de De Costa y Suranga 2012, Porro et al. 2012).

Potential accumulation of biomass and carbon under different types of forest management with impacts on REDD+ actions, mainly in multistrata agroforestry systems dominated by cacao (dashed lines) with successive crops every 30 years (A) and multistrata with fruit trees, timber species, and cacao with harvests every 25 years (B). Comparative trends for the most common forest plantations in the tropics and in the Amazon are indicated (continuous lines), such as those of Swietenia macrophylla (C) and Tectona grandis (D), both of which have a single harvest after 20 years (trends adapted from De Costa and Suranga 2012, and Porro et al. 2012).

almacenamiento estable de carbono y la agricultura continúa siendo unas de las principales causas de emisiones de carbono, deberían implementarse actividades REDD+ que vayan más allá de la conservación de bosques y reducción de emisiones; es decir, actividades que también contribuyan con el incremento de las reservas de carbono. Por este motivo, también se proponen cuatro urgentes actividades REDD+ para recuperar e incrementar las reservas de carbono y biodiversidad de bosques en la Amazonía dentro de un sistema de ordenación forestal (figura 5). Los barbechos mejorados a través de la sucesión controlada, son considerados después de un ciclo de agricultura migratoria como medida de restauración agrosucesional (cuadro 1).

Aún existen pocas experiencias de desarrollo de modelos agrosucesionales en la Amazonía, en particular sobre la sincronización y arreglos espaciales de las especies agroforestales con relación al tiempo de sucesión de especies nativas. En muchas regiones de la Amazonía, estos mode- los podrían iniciarse a partir de un barbecho tradicional, aprovechando la productividad de los cultivos anuales asociados con arbustos de ciclo corto, en la misma medida que se establecen especies frutales y maderables de ciclo largo, y manejando la cobertura de especies nativas (Villa et al. 2015). Sin embargo, estos investigadores discuten que en las comunidades indígenas de Amazonas, los sistemas de barbechos tienen especies nativas que crecen simultáneamente con cultivos tradicionales, pero sin una disposición espacial sistemática para un mejor uso equivalente de la tierra. Por esta razón, se propone un manejo especial para incrementar la densidad de plantas y diversidad de especies agroforestales, conocido también como "barbecho mejorado", a través del "enriquecimiento de especies". Este modelo SAF permitiría aumentar los rendimientos de la producción en comparación a monocultivos, bosques secundarios degradados, e incluso que plantaciones forestales, además del potencial para almacenar carbono en el suelo (figura 6).

Finalmente, es importante resaltar que el establecimiento de sistemas agrosucesionales, y alternativamente los sistemas agroforestales en áreas degradadas, tendrían un impacto importante para la mitigación ambiental desde el momento que estos sistemas sean permanentes por unidad de área (SAF de ciclos sucesivos, figura 6). Para esto es importante el manejo planificado de ciclos sucesivos de los componentes agroforestales, según sus fases fenológicas y productivas, a través de la subdivisión de áreas con diferentes cronosecuencias de desarrollo dentro del mismo sistema agroforestal (ordenación agroforestal). En este sentido, se puede planificar un futuro reemplazo de individuos de especies perennes senescentes, por individuos de fases de desarrollo inicial e intermedio establecidos sistemáticamente (figura 6). Este sistema tendría la ventaja de mantener la producción de especies anuales asociadas, en cada ciclo de manejo dentro de esas áreas subdivididas, hasta antes del cierre completo del dosel forestal. Adicionalmente, la restauración activa se puede implementar simultáneamente en áreas forestales degradadas dentro de la misma unidad de paisaje, a través de la nucleación con la transposición de banco de semillas provenientes de los bosques maduros circundantes. Esta acción REDD+ también puede implementarse en áreas agroforestales temporales o barbechos que han disminuido su capacidad productiva, pero que han incrementado la acumulación de materia orgánica en el suelo como para crear áreas de amortiguación dentro de la matriz forestal, o también, áreas de conección entre fragmentos y la matriz forestal (figura 5).

Así mismo, la restauración forestal en Amazonas como parte de las estrategias REDD+ debe trascender a la manipulación de estructura de comunidades ecológicas de acuerdo a sus potencialidades funcionales dentro del ecosistema, porque los servicios ecosistémicos que proporcionan los diferentes tipos de bosques de la región, especialmente el almacenamiento de carbono, también dependen 
Cuadro 1. Importancia de los diferentes tipos de manejo del bosque sobre las cinco actividades REDD + propuestas para la cuenca Amazónica: reducción de emisiones de deforestación (A) y degradación forestal (B), incremento de reservas de carbono (C), manejo sustentable del bosque (D), conservación de reservas de carbono (E). Se indican acciones que tienen una directa y fuerte influencia $(* *)$, y acciones con menor potencial (*). Adaptado de Kapos et al. (2012).

Importance of different forest management types on the five REDD + activities proposed for the Amazon basin: reduction emissions from deforestation (A) and forest degradation (B), carbon stocks enhancement (C), sustainable management of forest (D), conservation of carbon stocks (E). Actions that have direct and strong influence $(* *)$, and actions with lower potential (*) are indicated. Adapted from Kapos et al. (2012).

\begin{tabular}{|c|c|c|c|c|c|}
\hline \multirow{2}{*}{ Tipo de manejo del bosque y acciones potenciales para la conservación. } & \multicolumn{5}{|c|}{ Actividades REDD+ } \\
\hline & A & $\mathrm{B}$ & $\mathrm{C}$ & $\mathrm{D}$ & $\mathrm{E}$ \\
\hline \multicolumn{6}{|l|}{ Conservación de bosques } \\
\hline - Áreas naturales protegidas & $* *$ & $* *$ & $*$ & $*$ & $* *$ \\
\hline - $\quad$ Proteger bosques maduros y secundarios & $* *$ & $* *$ & $* *$ & * & $* *$ \\
\hline \multicolumn{6}{|l|}{ Agricultura sustentable } \\
\hline - Recuperación de áreas degradadas & * & $*$ & $* *$ & $*$ & \\
\hline - $\quad$ Sistemas Agroforestales & $*$ & $*$ & $*$ & $*$ & $*$ \\
\hline - Barbechos mejorados & $*$ & $* *$ & $*$ & $*$ & $*$ \\
\hline \multicolumn{6}{|l|}{ Manejo de recursos forestales } \\
\hline - Reducir tala selectiva & & $* *$ & & $*$ & \\
\hline - Ordenación forestal & $* *$ & $* *$ & $*$ & $* *$ & $* *$ \\
\hline \multicolumn{6}{|l|}{ Restauración forestal } \\
\hline - Recuperación de áreas degradadas & $*$ & $*$ & $* *$ & $*$ & \\
\hline - Restauración activa & & & $* *$ & & \\
\hline - Restauración pasiva & $*$ & $*$ & $* *$ & $*$ & \\
\hline
\end{tabular}

de una restauración funcional. Incluso, en los mismos sistemas agroforestales es posible seleccionar especies en función de la variación individual del tamaño de los árboles, que podría jugar un papel fundamental en el funcionamiento del ecosistema. Este tipo de enfoque no había sido considerado para SAF; sin embargo, recientemente Ali y Mattsson (2017) determinaron que la variación de la altura y diámetro de árboles tienen un efecto positivo sobre la biomasa aérea, seguido por un efecto no significativo de la diversidad de especies. Estos investigadores concluyen que la forma de manejo de la estructura de los SAF podría ser un enfoque efectivo para mejorar la productividad en estos sistemas, lo que a su vez dependerá del manejo integral de recursos forestales (cuadro 1).

\section{CONCLUSIONES}

Para incrementar la eficiencia en la aplicación de estrategias REDD+ en bosques de la Amazonía, es fundamental entender simultáneamente la relación de patrones y procesos de los ecosistemas forestales con el sistema socioecológico, a través del análisis de predictores biofísicos y antropogénicos, desde una escala local hasta una escala regional. Por lo tanto, además de conocer las característi- cas funcionales y estructurales de estos ecosistemas a través de predictores biofísicos que describen los gradientes climáticos y relación BEF, también se propone comenzar a comprender mejor el efecto multifactorial de predictores antropogénicos como la frecuencia, duración e intensidad de diferentes actividades asociadas con los cambios en el uso de la tierra, principalmente a través de la deforestación para la expansión de la frontera agrícola. Así mismo, evaluar simultáneamente los predictores antropogénicos que repercuten directamente sobre la demanda actual y futura de los bienes y servicios ecosistémicos, principalmente bajo escenarios dinámicos de migración, colonización y crecimiento poblacional. Estos predictores de evaluación de acciones REDD+ representan nudos críticos actuales para aumentar la eficiencia de conservación y manejo de ecosistemas forestales en la Amazonía. Por este motivo, se debe comenzar a evaluar la magnitud de los efectos que tienen los predictores de deforestación, especialmente los relacionados con la agricultura, para la integración de acciones REDD+ a través de la rehabilitación de áreas degradadas con sistemas agroforestales sucesionales y permanentes, así como con la aplicación de diferentes métodos de restauración (pasiva y activa) y de manejo integral de recursos forestales. 


\section{AGRADECIMIENTOS}

Agradecemos a los dos revisores anónimos que proporcionaron valiosos comentarios al manuscrito. El primer autor agradece a la Organización de los Estados Américanos (OEA) y el Fondo Mundial Ambiental (GEF) por apoyar el desarrollo de esta investigación.

\section{REFERENCIAS}

Aguiar APD, ICG Vieira, TO Assis, E Dalla-Nora, PM Toledo, RAO Santos-Junior, M Batistella, AS Coelho, EK Savaget, LEOC Aragão, CA Nobre, JPH Ometto. 2016. Land use change emission scenarios: anticipating a forest transition process in the Brazilian Amazon. Global Change Biology 22(5): 1821-1840. DOI: $10.1111 / \mathrm{gcb} .13134$

Ali A, E Mattsson. 2017. Individual tree size inequality enhances aboveground biomass in homegarden agroforestry systems in the dry zone of Sri Lanka. Science of the Total Environment 575: 6-11. DOI: 10.1016/j.scitotenv.2016.10.022

Angelsen A, D McNeill. 2013. Evolución de REDD+. In Angelsen A, M Brockhaus, WD Sunderlin, LV Verchot eds. Análisis de REDD+: Retos y opciones. Bogor, Indonesia. CIFOR. p. 35-56.

Aragão LEOC, B Poulter, J Barlow, L Anderson, Y Malhi, S Saatchi, O Phillips, E Gloor. 2014. Environmental change and the carbon balance of Amazonian forests. Biological Reviews 89(4): 913-931. DOI: 10.1111/brv.12088.

Arroyo-Kalin M. 2012. Slash-burn-and-churn: Landscape history and crop cultivation in pre-Columbian Amazonia. Quaternary International 249: 4-18. DOI: 10.1016/j. quaint.2011.08.004

Bennett AC, NG McDowell, CD Allen, KJ Anderson-Teixeira. 2015. Larger trees suffer most during drought in forests worldwide. Nature Plants 1(15139): 1-5. DOI: 10.1038/ nplants.2015.139

Bush MB, CH McMichael, DR Piperno, MR Silman, J Barlow, CA Peres, M Power, MW Palace. 2015. Anthropogenic influence on Amazonian forests in pre-history: An ecological perspective. Journal of Biogeography 42(12): 2277-2288. DOI: $10.1111 /$ jbi.12638

Brando PM, JK Balch, DC Nepstad, DC Morton, EP Francis, TC Michael, D Silvério, MN Macedo, EA Davidson, CC Nóbrega, A Alencar, BS Soares-Filho. 2014. Abrupt increases in Amazonian tree mortality due to drought-fire interactions. Proceedings of the National Academy of Sciences of the United States of America 111(17): 6347-6352. DOI: 10.1073/pnas.1305499111

Brienen RJW, OL Phillips, TR Feldpausch, E Gloor, TR Baker, J Lloyd, G Lopez-Gonzalez, A Monteagudo-Mendoza, Y Malhi, SL Lewis, R Vásquez Martinez, M Alexiades, E Álvarez Dávila, P Alvarez-Loayza, A Andrade, LEOC Aragão, A Araujo-Murakami, EJMM Arets, L Arroyo, GA Aymard, OS Bánki, C Baraloto, J Barroso, D Bonal, RGA Boot, JLC Camargo, CV Castilho, V Chama, KJ Chao, J Chave, JA Comiskey, F Cornejo Valverde, L da Costa, EA de Oliveira, A Di Fiore, TL Erwin, S Fauset, M Forsthofer, DR Galbraith, ES Grahame, N Groot, B Hérault, N Higuchi, EN Honorio Coronado, H Keeling, TJ Killeen, WF Laurance, S Laurance, J Licona, WE Magnussen, BS Marimon, BH
Marimon-Junior, C Mendoza, DA Neill, EM Nogueira, P Núñez, NC Pallqui Camacho, A Parada, G Pardo-Molina, J Peacock, M Peña-Claros, GC Pickavance, NCA Pitman, L Poorter, A Prieto, CA Quesada, F Ramírez, H RamírezAngulo, Z Restrepo, A Roopsind, A Rudas, RP Salomão, M Schwarz, N Silva, JE Silva-Espejo, M Silveira, J Stropp, J Talbot, H ter Steege, J Teran-Aguilar, J Terborgh, R Thomas-Caesar, M Toledo, M Torello-Raventos, RK Umetsu, GMF van der Heijden, P van der Hout, IC Guimarães Vieira, SA Vieira, E Vilanova, VA Vos, RJ Zagt. 2015. Long-term decline of the Amazon carbon sink. Nature 519: 344-360. DOI: 10.1038/nature14283

Cardinale BJ, JE Duffy, A Gonzalez, DU Hooper, C Perrings, P Venail, A Narwani, GM Mace, D Tilman, DA Wardle, AP Kinzig, GC Daily, M Loreau, JB Grace, A Larigauderie, DS Srivastava, S Naeem. 2012. Biodiversity loss and its impact on humanity. Nature 486: 59-67. DOI: 10.1038/nature 11148

Cumming GS, CR Allen. 2017. Protected areas as social-ecological systems: perspectives from resilience and socialecological systems theory. Ecological Applications 27(6): 1709-1717. DOI:10.1002/eap.1584

De Costa WAJM, HR Suranga. 2012. Estimation of carbon stocks in the forest plantations of Sri Lanka. Journal of the National Science Foundation of Sri Lanka 40(1): 9-41. http://doi. org/10.4038/jnsfsr.v40i1.4166

Diniz FH, MA Hoogstra-Klein, K Kok, B Arts. 2013. Livelihood strategies in settlement projects in the Brazilian Amazon: Determining drivers and factors within the Agrarian Reform Program. Journal of Rural Studies 32: 196-207. http:// dx.doi.org/10.1016/j.jrurstud.2013.06.005

D’Oliveira MV, EC Alvarado, JC Santos, JA Carvalho. 2011. Forest natural regeneration and biomass production after slash and burn in a seasonally dry forest in the Southern Brazilian Amazon. Forest Ecology and Management 261(9): 14901498. DOI: 10.1016/j.foreco.2011.01.014

Espindola GM, APD Aguiar, E Pebesma, G Câmara, L Fonseca. 2012. Agricultural land use dynamics in the Brazilian Amazon based on remote sensing and census data. Applied Geography 32(2): 240-252. DOI: 10.1016/j. apgeog.2011.04.003

Fauset S, MO Johnson, M Gloor, TR Baker, A Monteagudo, RJW Brienen, TR Feldpausch, G Lopez-Gonzalez, Y Malhi, H Steege, NCA Pitman, C Baraloto, J Engel, P Pétronelli, A Andrade, JLC Camargo, SGW Laurance, WF Laurance, J Chave, E Allie, PN Vargas, JW Terborgh, K Ruokolainen, M Silveira, GA Aymard, L Arroyo, D Bonal, H RamirezAngulo, A Araujo-Murakami, D Neill, B Hérault, A Dourdain, A Torres-Lezama, BS Marimon, RP Salomão, JA Comiskey, M Réjou-Méchain, M Toledo, JC Licona, A Alarcón, A Prieto, A Rudas, PJ van der Meer, TJ Killeen, B M Junior, L Poorter, RGA Boot, B Stergios, E Vilanova, FRC Costa, C Levis, J Schietti, P Souza, N Groot, E Arets, VC Moscoso, W Castro, EN Honorio, M Peña-Claros, C Stahl, J Barroso, J Talbot, IC Guimarães, G van der Heijden, R Thomas, VA Vos, EC Almeida, EA Davila, LEOC Aragão, TL Erwin, PS Morandi, EA de Oliveira, MBX Valadão, RJ Zagt, P van der Hout, P Alvarez, JJ Pipoly, O Wang, M Alexiades, CE Cerón, I Huamantupa-Chuquimaco, A Di Fiore, J Peacock, NCP Camacho, RK Umetsu, P Barbosa de Camargo, RJ Burnham, R Herrera, CA Quesada, J 
Stropp, SA Vieira, M Steininger, CR Rodríguez, Z Restrepo, AE Muelbert, SL Lewis, GC Pickavance, OL Phi1lips. 2015. Hyperdominance in Amazonian forest carbon cycling. Nature Communications 6: 6857. DOI: 10.1038/ ncomms 7857 .

Godar J, TA Gardner, EJ Tizado, P Pacheco. 2014. Actor-specific contributions to the deforestation slowdown in the Brazilian Amazon. Proceedings of the National Academy of Sciences of the United States of America 111(43):1559115596. DOI: $10.1073 /$ pnas.1322825111

Jagger P, K Lawlor, M Brockhaus, MF Gebara, DJ Sonwa, IAP Resosudarmo. 2013. Salvaguardas de REDD+ en el discurso de políticas nacionales y proyectos piloto. In Angelsen A, M Brockhaus, WD Sunderlin, LV Verchot eds. Análisis de REDD+: Retos y opciones. CIFOR, Bogor, Indonesia. p. 340-358.

Kapos V, WA Kurz, T Gardner. 2012. Impacts of forest and land management on biodiversity and carbon. In Parrota JA, C Wildburger, S Mansourian eds. Understanding Relationships between Biodiversity, Carbon, Forests and People: The Key to Achieving REDD+ Objectives. Vienna, Austria. A global assessment report IUFRO. p. 53-78.

Laurance WF, MA Cochrane, S Bergen, PM Fearnside, P Delamonica, C Barber, S DAngelo, T Fernandes. 2001. The future of the Brazilian Amazon. Science 291(5503): 438-439. DOI: $10.1126 /$ science.291.5503.438

Laurance WF. 2002. Predictors of deforestation in the Brazilian Amazon. Journal of Biogeography 29(5-6): 737-748. DOI: 10.1046/j.1365-2699.2002.00721.x

Lasky JR, M Uriarte, VK Boukili, DL Erickson, WJ Kress, RL Chazdon. 2014. The relationship between tree biodiversity and biomass dynamics changes with tropical forest succession. Ecology Letters 17(9): 1158-1167. DOI: 10.1111/ ele. 12322

Lapola DM, LA Martinelli, CA Peres, JPHB Ometto, ME Ferreira, CA Nobre, APD Aguiar, MMC Bustamante, MF Cardoso, MH Costa, CA Joly, CC Leite, P Moutinho, G Sampaio, BBN Strassburg, ICG Vieira. 2013. Pervasive transition of the Brazilian land-use system. Nature Climate Change 4: 27-35. DOI: $10.1038 /$ nclimate2056

Malhi Y, D Wood, TR Baker, J Wright, O Phillips, T Cochranek, P Meir, J Chave, S Almeida, L Arroyo, N Higuchi, TJ Killeen, SG Laurance, WF Laurance, S Lewis, A Monteagudo, DA Neill, P Núñez Vargas, N Pitman, CA Quesada, R Salomão, JNM Silva, A Torres Lezama, J Terborgh, R Vásquez Martínez, B Vinceti. 2006. The regional variation of aboveground live biomass in old-growth Amazonian forests. Global Change Biology 12(7): 1107-1138. DOI: 10.1111/j.1365-2486.2006.01120.x

Mitchard ETA, TR Feldpausch, RJW Brienen, G Lopez-Gonzalez, A Monteagudo, TR Baker, SL Lewis, J Lloyd, CA. Quesada, M Gloor, H ter Steege, P Meir, E Alvarez, A Araujo-Murakami, LEOC Aragão, L Arroyo, G Aymard, O Banki, D Bonal, S Brown, FI Brown, CE Cerón, VC Moscoso, J Chave, JA. Comiskey, F Cornejo, MC Medina, L Da Costa, FRC Costa, A Di Fiore, TF. Domingues, TL Erwin, T Frederickson, N Higuchi, EN Honorio Coronado, TJ Killeen, WF Laurance, C Levis, WE Magnusson, BS Marimon, BHM Junior, I Mendoza Polo, P Mishra, MT Nascimento, D Neill, MP Núñez Vargas, WA Palacios, A. Parada, G Pardo Molina, M Peña-Claros, N Pitman, CA Peres, L Poorter, A Prie- to, H Ramirez-Angulo, Z Restrepo Correa, A Roopsind, KH Roucoux, A. Rudas, RP Salomão, J Schietti, M Silveira, PF de Souza, MK Steininger, J Stropp, J Terborgh, R Thomas, M Toledo, A Torres-Lezama, TR van Andel, GMF van der Heijden, ICG Vieira, S Vieira, E Vilanova-Torre, VA Vos, O Wang, CE Zartman, Y Malhi, OL Phillips. 2014. Markedly divergent estimates of Amazon forest carbon density from ground plots and satellites. Global Ecology and Biogeography 23(8): 935-946. DOI:10.1111/geb.12168

Nepstad D, D McGrath, C Stickler, A Alencar, A Azevedo, B Swette, T Bezerra, M DiGiano, J Shimada, R Seroa da Motta, E Armijo, L Castello, P Brando, MC Hansen, M McGrath-Horn, O Carvalho, L Hess. 2014. Slowing Amazon deforestation through public policy and interventions in beef and soy supply chains. Science 344(6188): 1118-1123. DOI: $10.1126 /$ science. 1248525

Nogueira EM, AM Yanai, FOR Fonseca, PM Fearnside. 2015. Carbon stock loss from deforestation through 2013 in Brazilian Amazonia. Global Change Biology 21(3): 12711292. DOI: $10.1111 / \mathrm{gcb} .12798$

Oliver TH, MS Heard, NJB Isaac, DB Roy, D Procter, F Eigenbrod, R Freckleton, A Hector, CDL Orme, OL Petchey, V Proença, D Raffaelli, KB Suttle, GM Mace, B MartínLópez, BA Woodcock, JM Bullock. 2015. Biodiversity and Resilience of Ecosystem Functions. Trends in Ecology \& Evolution 30(11): 673-684. http://dx.doi.org/10.1016/j. tree.2015.08.009.

Peres CA, TA Gardner, J Barlow, J Zuanon, F Michalski, AC Lees, ICG Vieira, FMS Moreira, KJ Feeley. 2010. Biodiversity conservation in human-modified Amazonian forest landscapes. Biologycal Conservation 143(10): 2314-2327. DOI: 10.1016/j.biocon.2010.01.021.

Pérez CV, R Bilsborrow, B Torres. 2015. Income diversification of migrant colonists vs. Indigenous populations: Contrasting strategies in the Amazon. Journal of Rural Studies 42: 1-10. http://dx.doi.org/10.1016/j.jrurstud.2015.09.003

Perz SG, DL Skole. 2003. Social determinants of secondary forests in the Brazilian Amazon. Social Science Research 32(1): 25-60. http://dx.doi.org/10.1016/S0049089X(02)00012-1.

Piperno DR, C McMichael, MB Bush. 2015. Amazonia and the Anthropocene: What was the spatial extent and intensity of human landscape modification in the Amazon Basin at the end of prehistory? Holocene 25(10): 1-10. DOI: $10.1177 / 0959683615588374$

Poorter L, MT van der Sande, J Thompson, EJMM Arets, A Alarcón, J Álvarez-Sánchez, N Ascarrunz, P Balvanera, G Barajas-Guzmán, A Boit, F Bongers, FA Carvalho, F Casanoves, G Cornejo-Tenorio, FRC Costa, CV de Castilho, JF Duivenvoorden, L P Dutrieux, BJ Enquist, F FernándezMéndez, B Finegan, LHL Gormley, JR Healey, MR Hoosbeek, G Ibarra-Manríquez, AB Junqueira, C Levis, JC Licona, LS Lisboa, WE Magnusson, M Martínez-Ramos, A Martínez-Yrizar, LG Martorano, L C Maskell, L Mazzei, JA Meave, F Mora, R Muñoz, C Nytch, MP Pansonato, TW Parr, H Paz, EA Pérez-García, LY Rentería, J RodríguezVelazquez, DMA Rozendaal, AR Ruschel, B Sakschewski, B Salgado-Negret, J Schietti, M Simões, F L Sinclair, PF Souza, FC Souza, J Stropp, H ter Steege, NG Swenson, K Thonicke, M Toledo, M Uriarte, P van der Hout, P Walker, N Zamora, M Peña-Claros. 2015. Diversity enhances car- 
bon storage in tropical forests. Global Ecology Biogeography 24 (11): 1314-1328. DOI: 10.1111/geb.12364

Poorter L, F Bongers, TM Aide, AM Zambrano, P Balvanera, JM Becknell, V Boukili, PH S Brancalion, EN Broadbent, RL Chazdon, D Craven, JS de Almeida-Cortez, GAL Cabral, BHJ de Jong, JS Denslow, DH Dent, SJ de Walt, JM Dupuy, SM Durán, M M Espírito-Santo, MC Fandino, RG César, JS Hall, JL Hernandez-Stefanoni, CC Jakovac, AB Junqueira, D Kennard, SG Letcher, JC Licona, M Lohbeck, E Marín-Spiotta, M Martínez-Ramos, P Massoca, JA Meave, R Mesquita, F Mora, R Muñoz, R Muscarella, YRF Nunes, S Ochoa-Gaona, AA de Oliveira, E Orihuela-Belmonte, M Peña-Claros, EA Pérez-García, D Piotto, JS Powers, J Rodríguez-Velázquez, IE Romero-Pérez, J Ruíz, JG Saldarriaga, A Sanchez-Azofeifa, NB Schwartz, MK Steininger, NG Swenson, M Toledo, M Uriarte, $M$ van Breugel, $H$ van der Wal, MDM Veloso, HFM Vester, A Vicentini, ICG Vieira, T Vizcarra Bentos, GB Williamson, DMA Rozendaal. 2016. Biomass resilience of Neotropical secondary forests. Nature 530(7589): 211-217. DOI: 10.1038/nature16512

Porro R, RP Miller, MR Tito, JA Donovan, JL Vivan, R Trancoso, RF Van Kanten, JE Grijalva, BL Ramirez, AL Gonçalves 2012. Agroforestry in the Amazon Region: A Pathway for Balancing Conservation and Development. In Nair PKR, D Garrity eds. Agroforestry: The Future of Global Land Use. Advances in Agroforestry 9: 391-428. DOI 10.1007/97894-007-4676-3_20

Quesada CA, OL Phillips, M Schwarz, CI Czimczik, TR Baker, S Patiño, NM Fyllas, MG Hodnett, R Herrera, S Almeida, E Alvarez Dávila, AArneth, L. Arroyo, KJ Chao, N Dezzeo, T Erwin, A di Fiore, N. Higuchi, E Honorio Coronado, EM Jimenez, T Killeen, AT Lezama, G Lloyd, G López-González, FJ Luizão, Y Malhi, A Monteagudo, DA Neill, P Núñez Vargas, R Paiva, J Peacock, MC Peñuela, A Peña Cruz, N Pitman, N Priante Filho, A. Prieto, H Ramírez, A Rudas, R Salomão, AJB Santos, J Schmerler, N Silva, M Silveira, R Vásquez, I Vieira, J Terborgh , J Lloyd. 2012. Basinwide variations in Amazon forest structure and function are mediated by both soils and climate. Biogeosciences 9: 2203-2246. DOI: 10.5194/bg-9-2203-2012

Roosevelt AC. 2013. The Amazon and the Anthropocene: 13,000 years of human influence in a tropical rainforest. Anthropocene 4: 69-87. http://dx.doi.org/10.1016/j.ancene.2014.05.001.

Rodrigues-Filho S, R Verburg, M Bursztyn, D Lindoso, N Debortoli, AMG Vilhena. 2015. Election-driven weakening of deforestation control in the Brazilian Amazon. Land Use Policy 43: 111-118. http://dx.doi.org/10.1016/j.landusepol.2014.11.002

Strassburg BBN, A Kelly, A Balmford, RG Davies, HK Gibbs, A Lovett, L Miles, CDL Orme, J Price, RK Turner, ASL Rodrigues. 2010. Global congruence of carbon storage and biodiversity in terrestrial ecosystems. Conservation Letters 3(2): 98-105. DOI: 10.1111/j.1755-263X.2009.00092.x

Thompson ID, J Ferreira, T Gardner, M Guariguata, LP Koh, K Okabe, Y Pan, CB Schmitt, J Tylianakis. 2012. Forest biodiversity, carbon and other ecosystem services: relationships and impacts of deforestation and forest degradation. In Parrota J, C Wildburger, S Mansourian eds. Understanding Relationships between Biodiversity, Carbon, Forests and People: The Key to Achieving REDD+ Objectives. Vienna, Austria. A global assessment report IUFRO. p. 21-52

Verburg R, S Rodrigues-Filho, D Lindoso, N Debortoli, G Litre, M Bursztyn. 2014. The impact of commodity price and conservation policy scenarios on deforestation and agricultural land use in a frontier area within the Amazon. Land Use Policy 37: 14-26. http//dx.doi.org/10.1016/j.landusepol.2012.10.003

Villa PM, SV Martins, L Monsanto, SN Oliveira Neto, NM Cancio. 2015. La agroforestería como estrategia para la recuperación y conservación de reservas de carbono en bosques de la Amazonía. Bosque 36(3): 347-356. DOI: 10.4067/ S0717-92002015000300002

Wandelli VE, PM Fearnside. 2015. Secondary vegetation in central Amazonia: Land-use history effects on aboveground biomass. Forest Ecology Management 347:140-148. http:// dx.doi.org/10.1016/j.foreco.2015.03.020 